\title{
EchoGéo
}

15 | 2011

La Belgique, État prospère en décomposition au cœur de l'Europe du nord-ouest

\section{Les géographes, la coopération bilatérale et la recherche en partenariat}

Introduction

Alexis Sierra

\section{OpenEdition}

\section{Journals}

Édition électronique

URL : https://journals.openedition.org/echogeo/12318

DOI : $10.4000 /$ echogeo. 12318

ISSN : 1963-1197

Éditeur

Pôle de recherche pour l'organisation et la diffusion de l'information géographique (CNRS UMR 8586)

\section{Référence électronique}

Alexis Sierra, "Les géographes, la coopération bilatérale et la recherche en partenariat », EchoGéo [En ligne], 15 | 2011, mis en ligne le 09 juin 2011, consulté le 03 août 2021. URL : http:// journals.openedition.org/echogeo/12318; DOI : https://doi.org/10.4000/echogeo.12318

Ce document a été généré automatiquement le 3 août 2021.

EchoGéo est mis à disposition selon les termes de la licence Creative Commons Attribution - Pas d'Utilisation Commerciale - Pas de Modification 4.0 International (CC BY-NC-ND) 


\title{
Les géographes, la coopération bilatérale et la recherche en partenariat
}

\author{
Introduction
}

Alexis Sierra

1 Les conditions actuelles du financement et du développement de la recherche et de l'enseignement supérieur conduisent laboratoires et universités à constituer des montages institutionnels novateurs, permettant d'accéder à des ressources variées y compris en termes de réseaux d'échanges, de valorisation et de diffusion. En France, la création des unités mixtes de recherche entre laboratoires d'institutions différentes, le développement de l'agence nationale de la recherche avec ses financements croisés et la mise en place de fondations universitaires avec les entreprises privées, sont quelques exemples montrant l'obligation faite de travailler entre partenaires différents. Cependant, travailler en partenariat est loin d'être nouveau pour tous ceux qui ont dû développer enseignement et recherche à l'étranger. Si des cadres institutionnels et légaux ont été posés et se sont complexifiés, travailler avec des collègues d'autres organismes est aussi ancien que le travail de recherche lui-même. Pour le géographe, c'est une des dimensions essentielles du «travail de terrain » qui lui permet d'accéder au lieu d'observation, d'enquêtes, de collectes de données et en retour de diffuser le produit de sa recherche. Du point de vue français, on retient le nom de l'institution et du géographe français en méconnaissant souvent le partenaire étranger surtout s'il vient d'un pays du Sud. Cette logique a été quasi institutionnalisée par l'ANR qui ne reconnait pas les partenaires internationaux comme des membres de plein titre de l'équipe de recherche. Pourtant, les deux cas que présente ce dossier montrent l'importance et la valeur des collaborations tissées au fil des ans en Amérique latine, non seulement pour la recherche et l'enseignement dans cette région mais aussi (et parfois surtout) pour les Français. C'est ainsi une sorte de paradoxe : l'Etat français a développé une riche coopération internationale dont témoignent de nombreux instituts, mais les situations de collaboration sont assez mal connues en France. Ce dossier traite spécifiquement des coopérations entre Français et Latino-Américains. 
Nous retrouverions des ressorts communs à d'autres partenariats. L'intérêt ici est d'avoir affaire à une aire régionale qui a tissé des relations historiques différentes avec la France que ne l'ont faites l'Afrique ou l'Asie. Ici le Français n'est pas un ancien colon, même si comme Européen il peut être assimilé aux anciens colonisateurs. La latinité, la parenté idéologique et intellectuelle contre les dictatures ont pu créer des affinités instinctives qui ne doivent pour autant pas masquer les incompréhensions et surtout pas le désir des partenaires latino-américains d'être traité non en pays du Sud mais à égalité, même quand ils ont conscience des faiblesses de leurs moyens.

2 Comme nous allons le voir, ces partenariats à l'étranger sont souvent anciens. L'expérience montre même que ce sont des relations tissées sur plusieurs décennies qui permettent de construire des programmes de recherche et d'enseignement utiles aux deux parties. Robert D'Ercole souligne ainsi qu'une génération de Quiténiens a été sensibilisée et formée par des Français à des thématiques de recherche qui se sont révélées novatrices à partir de ces terrains-là. Hervé Théry rappelle que la coopération universitaire entre géographes du Brésil et de la France doit à des relations remontant aux années trente. Nous ne pouvons ainsi manquer de rappeler le développement de l'école française de géographie au Brésil plus particulièrement construite au lendemain de la Seconde guerre mondiale, notamment avec Pierre Monbeig. Ces échanges se sont poursuivis malgré la dictature militaire à travers des figures d'exil comme Milton Santos, maintenues et renforcées avec le retour de la démocratie aussi bien par les instituts de recherches (CNRS, Orstom/IRD) que par les Universités. Profitons de ce moment pour souligner combien ces relations scientifiques et académiques francobrésiliennes tendent aujourd'hui à se développer et à se diversifier à la demande des deux parties ${ }^{1}$.

3 Le temps, la mise en place de réseaux sur la durée, la reconnaissance mutuelle du travail effectué, la régularité des échanges, la présence sur le terrain sont des conditions essentielles à la mise en place de coopérations solides. Et pour en surmonter les difficultés! En effet, comme l'évoque Robert D'Ercole, la recherche en coopération doit faire face à une altérité à tous les niveaux : inégalités des conditions de travail, points de vues à rapprocher, environnements politique et administratif distincts. Ce géographe rappelle ainsi que les changements au sein des institutions à l'occasion des alternances politiques ou de réformes législatives représentent une épée de Damoclès dans ce travail en partenariat: les interlocuteurs d'aujourd'hui ne seront plus forcément ceux de demain et il faut dans ce cas sans cesse remettre le métier à l'œuvre pour tisser de nouvelles relations. L'exemple de la coopération avec la municipalité équatorienne avec une continuité administrative sur plus de vingt ans (et malgré le changement de maire) en montre les bénéfices. Les relations interpersonnelles, et souvent informelles, jouent un rôle déterminant dans la mise en place de partenariats durables. C'est la confiance tissée entre des personnes travaillant ensemble depuis longtemps qui a permis de surmonter les différences initiales et de créer des collaborations inédites, y compris avec des institutions qui n'ont pas initialement un rayonnement international comme l'indiquent les échanges entre universités françaises et brésiliennes.

Car ce qui est posé à travers la recherche et l'enseignement en partenariat, c'est bien la transmission d'un savoir construit en commun. Transmission sur le terrain, afin que ce qui est effectué ne serve pas qu'au géographe français en France. Transmission en France, pour que ces expériences soient reconnues et fassent évoluer la recherche et la 
formation en France. C'est pourquoi une grande ouverture est nécessaire, qui n'exclut pas a priori des partenaires qui ne seraient pas strictement académiques. La relation entre la recherche et l'expertise d'une part, la recherche et la formation (y compris professionnelle) d'autre part sont ainsi présentées dans toutes leurs richesses et leurs potentialités. La formation en particulier est un levier pour construire des relations de long terme; c'est pourquoi la mise en place de bourses permetttant aux étudiants étrangers de venir en France et aux étudiants français de découvrir d'autres terrains et d'autres pratiques s'est révélé un outil fondamental dans la coopération scientifique et universitaire.

5 Pour appréhender ces thèmes et composer ce dossier nous avons recueilli le témoignage de Robert D'Ercole, géographe, directeur de recherche de l'Institut de Recherche pour le développement, et l'analyse de Hervé Théry, géographe, directeur de recherche au CNRS. Le premier présente une expérience de recherche en coopération qui s'étale depuis le milieu des années quatre-vingt, essentiellement en Amérique latine et caraïbe. Elle couvre un large éventail de partenariats : universitaire, instituts de recherches, municipalités, organismes gouvernementaux, bureaux d'expertises, ONG. L'article de Hervé Théry présente une analyse géographique et quantitative d'une coopération internationale universitaire qui puise ses racines sur plusieurs décennies. Nous avons voulu présenter deux démarches différentes mais toutes deux réflexives et montrant la richesse qu'il y a à s'ouvrir à des coopérations internationales, même quand le géographe semble peiner à être reconnu comme tel, même si de nombreux obstacles, politiques, administratifs, culturels sont à franchir.

6 Dans les deux cas, la place du géographe est en effet posée. On remarquera que les sciences exactes, de la vie et de la Terre sont privilégiées dans ces coopérations laissant la géographie à une place seconde. Cependant, l'expérience de Robert D'Ercole montre que c'est également par le biais de relations avec les sciences de la terre que la géographie s'affirme et montre son apport, ce que pourrait confirmer Hervé Théry par sa présentation des thèmes d'enseignement développés entre géographes français et brésiliens, dans laquelle les études physiques et environnementales ont eu une place importante. Dans la même veine, on pourrait souligner que la ville tant comme terrain que comme objet d'étude est particulièrement présente dans les expériences présentées. C'est d'ailleurs devenu un enjeu scientifique majeur aussi bien autour de la question des risques (les villes concentrant les mises en exposition à de multiples menaces) qu'autour de l'aménagement de métropoles et de leur rôle dans la mondialisation (voir le dossier présenté sur le Forum Urbain Mondial de Rio en 2010). Le géographe y trouve plus facilement une reconnaissance en apportant la transversalité nécessaire à la compréhension d'un territoire que le politique doit gérer. Rappelons enfin que la reconnaissance de la géographie est loin d'être la même au Brésil, où une école de pensée s'est construite au long du $\mathrm{XX}^{\mathrm{e}}$ siècle, et dans les pays andins. Nonobstant, y compris dans ces derniers, l'intérêt de la géographie s'affirme notamment par la nécessité d'élaborer des politiques de planification des territoires. Le géographe rejoint l'élu puisque les deux doivent raisonner sur un territoire qui est également un cadre d'action. De ce point de vue, la réflexion du général Paco Moncayo, maire de Quito, à Robert D'Ercole est savoureuse quand il estime que finalement les géographes raisonnent comme les militaires, en hiérarchisant les lieux, en aidant à fixer des priorités d'intervention sur le territoire. Une remarque qui donne un réel 
écho à ce que Yves Lacoste démontra sur les relations entre géographie et politique et qui peut être un levier pour démontrer l'utilité d'une coopération bilatérale.

\section{NOTES}

1. Cet intérêt réciproque se manifeste par plusieurs voies. De manière directe, comme l'illustre parmi d'autres exemples, le Forum Urbain Mondial en 2010 (EchoGéo ${ }^{\circ} 12$ ). Cet événement a mis en présence des collègues brésiliens avec une délégation française particulièrement nombreuse (la deuxième représentée après les Etats-Unis). Indirectement, les partenariats anciens construits sur d'autres terrains latino-américains favorisent la relation franco-brésilienne. Ainsi, les géographes français travaillant avec ceux des pays andins sont amenés à se rapprocher des collègues brésiliens pour étudier les espaces frontaliers et amazoniens.

\section{AUTEUR}

\section{ALEXIS SIERRA}

Alexis Sierra est maître de conférences en géographie à l'université Cergy-Pontoise-IUFM.

alexisierra2001@yahoo.fr 